\title{
Electrical transport studies of quench condensed Bi films at the initial stage of film growth: Structural transition and the possible formation of electron droplets
}

\author{
M. M. Rosario and Y. Liu \\ Department of Physics, The Pennsylvania State University, University Park, PA 16802
}

(November 21, 2018)

\begin{abstract}
The electrical transport properties of amorphous Bi films prepared by sequential quench deposition have been studied in situ. A superconductor-insulator (S-I) transition was observed as the film was made increasingly thicker, consistent with previous studies. Unexpected behavior was found at the initial stage of film growth, a regime not explored in detail prior to the present work. As the temperature was lowered, a positive temperature coefficient of resistance $(\mathrm{d} R / \mathrm{d} T>0)$ emerged, with the resistance reaching a minimum before the $\mathrm{d} R / \mathrm{d} T$ became negative again. This behavior was accompanied by a non-linear and asymmetric $I-V$ characteristic. As the film became thicker, conventional variable-range hopping $(\mathrm{VRH})$ was recovered. We attribute the observed crossover in the electrical transport properties to an amorphous to granular structural transition. The positive $\mathrm{d} R / \mathrm{d} T$ found in the amorphous phase of Bi formed at the initial stage of film growth was qualitatively explained by the formation of metallic droplets within the electron glass.
\end{abstract}

73.50.-h, 74.40.+k

\section{INTRODUCTION}

Quench condensed films, prepared by depositing metal vapor directly onto substrates held at liquid helium temperatures, have played important role in elucidating the effects of interactions, disorder, and geometrical constraints on the properties of an electronic system. Films made in this manner are conducting at thicknesses as low as $d \approx 10 \AA$, 2 making it a two-dimensional (2D) disordered system. Quench condensed films have been used in numerous experimental studies of superconductivity, localization 1 and their interplay. More recently, it has played an important role in the study of quantum phase transitions, such as the 2D superconductor-insulator (SI) and metal-insulator (M-I) transitions.

Physics in a strongly disordered system is dominated by electron-electron interactions. These interactions result in the formation of a Coulomb gap, namely a reduction in the single-particle density of states (DOS) near the Fermi energy 6 leading to a medification of variablerange-hopping (VRH) conduction. 0 The temperature dependence of electrical conductivity in the $\mathrm{VRH}$ regime is given by $\sigma(T)=\sigma_{\mathrm{o}} \exp \left[-\left(T_{\mathrm{o}} / T\right)^{a}\right]$, where $\sigma$ is the electrical conductivity, $T$ is temperature, $\sigma_{\mathrm{o}}$ and $T_{\mathrm{o}}$ are constants, and $a=1 / 3,1 / 4$ in non-interacting $2 \mathrm{D}$ and $3 \mathrm{D}$ systems, respectively. The introduction of Coulomb interactions results in $a=1 / 2$, regardless of the dimensionality, with the constant $T_{\mathrm{o}}$ given by $T_{\mathrm{o}}=\mathrm{e}^{2} /\left(\mathrm{k}_{\mathrm{b}} \epsilon \xi_{\mathrm{L}}\right)$, where $\epsilon$ is the dielectric constant and $\xi_{\mathrm{L}}$ is the localization length.

The Coulomb gap problem can be mapped onto an Ising spin glass model with $1 / r$ antiferromagnetic interactions in a random field, suggesting that finitetemperature glass transition may be present. 12 An order parameter, similar to the Edwards-Anderson order parameter used for spin glass systems, was proposed for the electron glass in an attempt to find a glass transition. Though a non-zero value of the order parameter was found, critical behavipr was absent in the numerical studies. Subsequent work 10 revealed that a glass transition is not present at mon-zero temperatures, similar to 2D Ising spin glasses. 13

Experimental studies of the electron glass were carried out originally on lightly doped semiconductors.14 Recently field-effect experiments on strongly diserdered systems, 15,16 including quench condensed films, 17 have revealed glassy behavior (but not critical behavior associated with a glass transition). In particular, effects such as slow relaxation, hysteresis, memory andaging are documented in quench condensed $\mathrm{Bi}$ and $\mathrm{Pb} .17$ In addition, an observed minimum in the conductance as a function of gate voltage was proposed to be related to the opening of the Coulomb gap in the DOS.18 Interestingly, though the conductance for the films was found to fit the general hopping form, theyalue for $a$ obtained from the fitting was close to $0.8,17,19$ a dependence not given by any VRH theory.

Recently, in situ scanning tunneling microscopy (STM) studies on granular quench condensed films of $\mathrm{Ay}$ and $\mathrm{Pb}$ revealed an unexpected scenario for film growth.2d It appears that a uniform, homogeneously disordered phase formed during the initial depositions. This precursor phase was found to avalanche into an islanded structure as the thickness reached a critical value. Results obtained on both $\mathrm{Au}$ and $\mathrm{Pb}$ films indicate that the observed structural evolution may be general, independent of substrate material and film preparation procedure. A similar amorphous precursor phase was found in in situ Ramanscattering measurements on Bi films deposited onto carbon substrates at 110K 21 The Raman spectra indicated a distinct local structural transition from amorphous-like 
to nanocrystalline clusters as the film thickness increased across a critical thickness of $\sim 8 \AA$.

These uniform, amorphous films should also form at the initial stage of film growth in Bi films quench condensed at liquid helium temperatures, even though the local amorphous to crystalline structural transformation may be incomplete because of the reduced substrate temperature. The electrical transport properties of these films at the initial stage of film growth, which have never been studied as the regular sized films are too resistive to measure, are of great interest. Transport measurements on films of macroscopic size, taken simultaneously with the in situ STM studies, showed that the onset of conductivity occurred when the films were seen to have two nearly complete layers of grains, having formed electrically connected multigrain clusters. The undetectable electrical conductivity of the initial amorphous precursor phase on a macroscopic scale suggests either electron localization at an atomic scale because of the strong disorder,22 or structural discontinuity at a large length scale even though electrons within each connected amorphous region are not necessarily strongly localized. The prevailing view appears to favor the former scenario.

We have studied the electrical transport properties of quench condensed $\mathrm{Bi}$ films in this precursor regime. While conventional glassy behavior was found in these thinnest films, an unexpected positive temperature coefficient of resistance $(\mathrm{d} R / \mathrm{d} T>0)$, accompanied by a non-linear and asymmetric current-voltage $(I-V)$ characteristic, was observed in the initial stage of film growth. As more Bi was deposited, this state was found to enter a more familiar state characterized by VRH with a Coulomb gap. This crossover in electrical transport properties appeared to coincide with an amorphous to granular structural transition in ultrathin Bi reported previously 20 The positive $\mathrm{d} R / \mathrm{d} T$ found in the initial films is proposed to be a result of the formation of electron droplets within an electron glass state.

\section{SAMPLE PREPARATION AND MEASUREMENTS}

Films were prepared by quench deposition in a ${ }^{3} \mathrm{He}$ cryostat.23 A glazed alumina (amorphous $\mathrm{Al}_{2} \mathrm{O}_{3}$ ) substrate, held at liquid helium temperatures during the evaporation of $\mathrm{Bi}$, was used. An amorphous Ge underlayer, previously used to help grow an electrically homogeneous film, was not evaporated onto the substrate prior to the Bi evaporation. Current biased d.c. electrical transport measurements were taken in situ after each evaporation. A set of films was created by sequential deposition. To ensure that any possible annealing was avoided, all films were kept at temperatures lower than $10 \mathrm{~K}$, and bias currents of less than $1 \mathrm{nA}$ were used.

A major difficulty in studying the electrical transport properties of the films at the initial stage of growth is the extremely high resistivity, resulting in an unmeasurable resistance for samples of conventional size. This difficulty can be circumvented by preparing films of extremely short length but relatively large width, which can be conveniently done using a 2-point probe configuration. An undesired consequence of this approach is the somewhat uncontrolled contact resistance associated with 2-point measurements. We have addressed this experimental issue in two ways. First, we made an effort to design the sample configuration that would minimize the contact resistance. Second, we studied samples of different lengths, so that the contact resistance can be inferred from a scaling analysis.

In the present work, $100 \AA$ thick $\mathrm{Au}$ measurement leads were pre-patterned onto the substrate. Films were deposited across a narrow gap between two $\mathrm{Au}$ contacts and atop the entire $\mathrm{Au}$ electrode. Since the $\mathrm{Au}$ film is significantly more conductive than the initial Bi films, the measured resistance is dominated by the resistance of the Bi film in the gap and the contact resistance. For the latter, the following steps were taken to minimize its magnitude. The gap was prepared using a shadow mask, resulting in a smooth and gradual edges, as revealed by atomic force microscope (AFM) study. The combination of the gradual slope and the good conductivity of the $\mathrm{Au}$ thin film is expected to minimize the contact resistance, as appeared to be the case experimentally (see below). We studied two film sequences of lengths $2.5 \mu \mathrm{m}$ and 35 $\mu \mathrm{m}$, respectively, both $0.5 \mathrm{~mm}$ in width. A schematic of the sample configuration is shown in the inset of Fig. 1.

Another experimental difficulty in measuring films at the initial stage of film growth is the exceedingly long equilibrium times, due to both the large RC constant and intrinsic glassy behavior. We monitored the time response of the voltage for all films which exhibited long settling times. All values shown are the limiting values of the voltage rise after adequate time was allowed for the measurement to stabilize. The glassy state previously observed in strongly disordered systems are often characterized by extremely long, non-exponential relaxation times.16.17 Similar behavior was observed in the present set of films in the region where $\mathrm{R}>10^{7} \Omega$ (corresponding to sheet resistances of $R_{\square}>10^{9}$ ), as shown in Fig.1. The voltage response can be fit to a stretched exponential of the form $V(t)=V_{\mathrm{o}}\left(1-\exp \left[-(t / \tau)^{\alpha}\right]\right)$, where $V_{\mathrm{o}}$ is the limiting value of the voltage, $\tau$ the time constant, and $\alpha$ the exponential constant. The time constants obtained were twice as large as expected from the RC constant of the measurement system.

\section{EXPERIMENTAL RESULTS}

In addition to long relaxation times, films at the initial stage were found to have a non-linear, asymmetric $I-V$ characteristic, as seen in Fig. 2. The asymmetry and non-linearity were more pronounced at lower tempera- 
tures and smoothly evolved to simple ohmic behavior as temperature was increased. Non-linearity, in itself, is not unexpected in systems dominated by VRH conduction. However, an analysis of the data using theoretically predicted dependences of conductance on electric field in the VRH regime 24 was inconclusive, as a satisfactory fit was not found. The asymmetry about zero current bias in both magnitude and curvature seen in the $I-V$ curves has not been theoretically predicted, but may be understood qualitatively in a model proposed here (see below).

Given the nature of the $I-V$ characteristic, d.c. resistances were calculated by measuring the voltage across the sample at a fixed current bias of $+20 \mathrm{pA}$. The voltage at zero bias current is subtracted from this value to account for offset voltages, a common practice in electrical transport measurements. The resistance characteristic at negative current bias is qualitatively similar to that measured with a positive bias.

The resistance as a function of temperature $\mathrm{R}(T)$ of the $2.5 \mu \mathrm{m}$ long film, at a thickness $d$ of $12.5 \AA$, is given in Fig. 3. Inset a) shows a Zabrodskii plot 25 of the data, where the function $\ln [-d(\ln R) / d(\ln T)]$ is plotted against $\ln T$. If the resistance is described by the hopping conduction formula, $R=R_{\mathrm{o}} \exp \left[\left(T_{\mathrm{o}} / T\right)^{a}\right]$, we expect a straight line in such a plot. The hopping exponent $a$ would correspond to the slope of the line. A least squares line fit yielded a value $a=0.49 \pm 0.07$, consistent with VRH with Coulomb interactions. A fit of the data specifically to this form is given in inset $\mathrm{b}$ ). The localization length is estimated to be $\xi_{\mathrm{L}} \approx 80 \AA$, assuming that the dielectric constant of the film is that of the substrate. Similar fits to simple activated behavior $(a=1)$ and non-interacting VRH conduction ( $a=1 / 3,1 / 4)$ were unsatisfactory. The temperature dependence of the conductivity and the slow voltage response indicate that these films can be characterized as being in an electron glass state.

As indicated in Fig. 3 inset b), the resistance deviated from VRH (with interactions) at low temperature. The temperature at which the deviation began was close to the temperature at which the $I-V$ characteristic exhibited asymmetric and non-linear behavior. In thinner films, the temperature above which VRH was observed increased, with the resistance deviation persisting to increasingly higher temperatures. In the thinnest films, standard hopping conduction was no longer observed within the temperature range studied $(<10 \mathrm{~K})$. In addition, a non-linear and asymmetric $I-V$ characteristic was present over the whole temperature range.

Remarkably, in these thinnest films, the low temperature deviation developed into a positive temperature coefficient of resistance, i.e. $\mathrm{d} R / \mathrm{d} T>0$. Fig. 4 a $\mathrm{a}-\mathrm{d}$ shows the $\mathrm{R}(T)$ of films in this regime in detail. The positive $\mathrm{d} R / \mathrm{d} T$ did not extend down to $T=0$, but instead reached a resistance minimum and recovered insulating behavior in the limit of zero temperature. The resistance at intermediate temperatures showed an almost linear temperature dependence. In contrast, the low temperature insulating behavior was found to rise dramatically with decreasing temperature. The precise temperature dependences in these two regimes were difficult to determine due to the small temperature range available for data fitting.

An examination of behavior in films of different thicknesses revealed that the positive $\mathrm{d} R / \mathrm{d} T$ persisted to higher temperatures with increasing disorder, as seen in Figs. 4a-d. In contrast, the resistance minimum occurred at $T \approx 2.5-3 \mathrm{~K}$, for all films which exhibited a positive $\mathrm{d} R / \mathrm{d} T$, regardless of film thickness. This feature disappeared at $d=12.0 \AA$.

The evolution of the film resistance with respect to film thickness is shown in Fig. 5. For $d \geq 12.0 \AA$, a striking departure from the behavior of the thinnest films was seen in the resistance characteristic. Although a non-linear and asymmetric $I-V$ characteristic was still observed at the lowest temperatures, a resistance minimum was no longer found. The films exhibited an insulating character over the whole temperature range $(T<8 \mathrm{~K})$. For films with $d>12.5 \AA$, the $I-V$ curves displayed ohmic behavior over the whole temperature $(T<8 \mathrm{~K})$ and current range $(I<100 \mathrm{pA})$. As more material was added the resistance was observed to enter a region where the resistance could not be fitted to any theoretically predicted dependence.

As the film was made thicker, familiar behavior for granular ultrathin films of superconducting metals, including a superconductor-insulator (S-I) transition was found, similar to what has been previously observed.26 On the insulating side of the transition, the resistance displayed hopping conduction characteristics at higher temperatures, but leveled off at low temperatures. The level-off phenomenon has been widely observed in ultrathin insulating films.2627 Further increases in thickness leads to a weakly localized regime where the resistance has a $\ln T$ dependence.

The kink in the $\mathrm{R}(T)$, below which a more rapid rise in resistance was seen, has been attributed to the opening of the superconducting energy gap in individual grains at a transition temperature $T_{\mathrm{co}} 28$ As a result, the activation energy for single electron hops between grains is the sum of the charging and the superconducting pairing energy.28 30 As more material was deposited, the kink developed into a resistance minimum, a well kpown phenomenon of quasi-reentrant superconductivity.28 31 In this case, although individual grains become superconducting with zero resistance which causes a resistance drop at $\mathrm{T}=T_{\mathrm{co}}$, the phases of these grains do not establish a long-range coherence. 32 Quantum fluctustions drive the film insulating at lower temperatures. 33 The observed re-entrant behavior, which has become the hallmark for granular superconducting films, indicates that the Bi films prepared in the present study were granular as well.

As seen in Fig. 5, the evolution of the resistance as a function of film thickness is remarkably systematic: in the thinnest films, the positive temperature coefficient of resistance becomes less distinct in relatively thicker films; at intermediate thicknesses the films develop stan- 
dard VRH behavior at higher temperatures, as well as with increasing thickness; finally, further increases in film thickness result in an S-I transition, consistent with previous studies. Qualitatively similar behavior was also observed in studies of the $35 \mu \mathrm{m}$-long film.

It is important to examine the issue concerning the contact resistance between the $\mathrm{Bi}$ and the $\mathrm{Au}$ contacts. A comparison of resistances of the co-deposited $2.5 \mu \mathrm{m}$ and $35 \mu \mathrm{m}$-long films, as well as values of contact resistance, are shown in Table 1. Films thinner than $11.0 \AA$ have contact resistances that are neglible compared to the film resistance. For films thicker than $15.5 \AA$, the contact resistance is found to be a significant fraction of the film resistance. However, as noted above, the behavior is strikingly similar to what was previously observed in this system using a 4-point configuration. This indicates that, despite the 2-point configuration, the intrinsic nature of the film is reflected in the measurement. This is possible if the contact resistance, though large, is temperature independent. Finally, films of intermediate thicknesses were found to not scale, and a value for the contact resistance could not determined; this issue will be discussed in the following section.

\section{AMORPHOUS TO GRANULAR STRUCTURAL TRANSITION}

The intriguing crossover of the resistance characteristic with respect to film thickness observed at around $12.0-14.0 \AA$ in quench condensed Bi films has raised the question concerning its physical origin. Below we argue that this crossover may be related to film growth models adyanced by in situ Raman studies on quench condensed $\mathrm{Bi}, 21$ and STMresults obtained on quench condensed $\mathrm{Au}$ and $\mathrm{Pb}$ films 20

The abrupt disappearance of the resistance minimum in the thinnest films, which marked a qualitative change in the resistance characteristic, appears to point to a scenario similar to the avalanche model proposed to explain the in situ STM observations. Fig. 6] shows the sheet resistance, $R_{\square}$, at $\mathrm{T}=8 \mathrm{~K}$ for the entire set of films. Within the range $11.5 \AA<d<14.0 \AA, R_{\square}$ decreased particularly rapidly with increasing thickness, indicating that the films underwent a qualitative change in this thickness range.

The behavior seen in Fig. 6 indicates the existence of two distinctly different regimes, with a rapid crossover region observed over a change in film thickness of only around $2 \AA$. In these two regions, the thickness dependences of $R_{\square}$ were similar, exhibiting a relatively slow change with increasing thickness in comparison with the transition region, though the values of $R_{\square}$ in the two limits $(d<11.5 \AA$ and $d>14.0 \AA)$ differ by more than 5 orders of magnitude. Interestingly, at $t=13.0 \AA$, a thickness corresponding to the middle of the transition region, the film resistance does not follow any theoretically predicted dependence and, instead, appears as a boundary between the high resistance and low resistance regions. The significant difference in magnitude of the resistivity, the sharp transition, as well as the distinct difference in character between the two regimes may be explained by a change in the film's structure as the thickness is increased.

Such a change is further supported by the lack of scaling between the $2.5 \mu \mathrm{m}$ - and $35 \mu \mathrm{m}$-long film in and near the transition region (see Table 1). As the films undergo the transition, a characteristic length determines the films behavior. Films whose size is larger than this length should scale, whereas those smaller should not. The rapidly changing resistance found in this thickness range suggests that this length is strongly thickness dependent. As the films moved farther into the low resistance region, they were again found to scale, indicating the end of the transition, and the emergence of a stable film structure.

Within this structural transition scenario, the film develops from a uniform to granular film at a critical thickness $d \approx 11.5 \AA$. Assuming that the resistance in the granular film after the transition was dominated by intergrain electron hopping, further material depositions serves to decrease the tunnel barrier strength between grains, increasing the conductivity. Therefore, the resistance of the films would depend on thickness more sensitively in just-formed granular films, and is less sensitive in the relatively thicker films after a path of grains across the film is already established, consistent with experimental observation.

In addition, within each individual grain, a local amorphous to crystalline structural transformation occurs, similar to that observed in in situ Raman-scattering measurements in Bi films deposited at 110K. In that study, the transition from an amorphous, disordered structure to a rhombohedral phase was found between $7.0 \AA$ and 8.5 $\AA$. The presence of such crystalline-like grains is supported by an observed $T_{\text {co }}$ of $2.5-3.0 \mathrm{~K}$ near the S-I transition. Similar values of $T_{\text {co }}$ were found in nanocrystalline Bi clusters. 34 However, given the lower deposition temperatures used in the present study and, therefore, the lower thermal energy available for Bi atom diffusion, a larger critical thickness for the local amorphous to crystalline transition $(d \approx 11.5 \AA)$ would be natural. Furthermore, the local transition in the present films could be incomplete for the same reason, resulting in grains that are between amorphous and crystalline in nature. We refer to this phase as quasi-crytalline. The thickness dependence of $T_{\mathrm{co}}$, i.e. that $T_{\mathrm{co}}$ increases with increasing thickness to a maximum value of $6.5 \mathrm{~K}$, provides further support that the grains after the structural transition retain a substantially amorphous character.

The proposed phase diagram for this transition is shown in the inset of Fig. 6. As temperature increases, an additional previously studied transition to a nanocrystalline phase 2 is expected (not indicated in the schematic). 


\section{POSSIBLE DROPLET FORMATION IN THE ELECTRON GLASS}

Another question raised by the present work is the nature of the electrical transport properties of the amorphous films found at the initial stage of film growth. It is remarkable that the temperature at which the resistance starts to rise in these films coincides with the $T_{\text {co }}$ of the locally superconducting, granular films near the S-I transition, as seen in Fig. 5. This observation can be understood if we assume that in the thinnest films, which should be homogeneously disordered over a large area, there may be locally superconducting regions (superconducting droplets). The resistance increase at low temperature would then be related to the opening of the energy gap in the superconducting droplets, similar to the granular superconducting film. The question is whether these droplets could indeed form in these strongly insulating films.

We note that a droplet state was proposed in a theoretical study of the 2D interacting, but highly mobile, electron system 36 Motivated by the recent observations of the 2D MIT,37 it was suggested that such a system is unstable against phase separation into a high-density Fermi gas ("liquid" phase) and a low density insulating Wigner crystal ("gas" phase). The formation of a twophase coexistence region, a droplet state, was shown to be favored by increasing potential disorder. Similarly, droplets might have begun to form in these amorphous films at a characteristic temperature, growing in size as temperature decreases. The resistance is determined by single electron hops between droplets. As the distance between droplets decrease with decreasing temperature, as does the resistance. This results in the observed deviation from VRH as well as the positive $\mathrm{d} R / \mathrm{d} T$.

The formation of these droplets minimizes the Coulomb repulsion energy of the system in order to be stable. However, the entropy of the system decreases as a result. At lower temperatures, the energy change is likely sufficient to compensate for the loss of entropy. At higher temperatures, the entropy term becomes more important and droplet formation may be less favored, resulting in a "gas" (uniform electron glass) state with insulating behavior, consistent with the thickness dependence of the onset of the positive $\mathrm{d} R / \mathrm{d} T$.

This picture can also qualitatively account for the asymmetry in the $I-V$ characteristic. Nucleation sites for the droplets are highly dependent on the potential disorder landscape present in the system, which is, in turn, dependent on the applied electric field. Taking into account that the films' left and right interfaces are not identical, the effective applied field need not be symmetric with respect to field direction. It follows that the spatial distribution of nucleation sites are unidentical, and the lowest resistance path is not expected to be the same for different field polarities.

The formation of such droplets may be natural if we consider the one-to-one correspondence of the Coulomb glass to the Ising spin glass. A phenomenological scaling theory of the ordered phase of short-range Ising spin glasses was developed in terms of droplet excitations. 35 In this model, low lying droplet excitations, consisting of connected clusters of spins reversed from the ground state, dominate the physics of the spin glass. A similar phenomena would not be unexpected in the electron glass.

\section{SUMMARY}

We have measured the electrical transport properties of quench condensed Bi films deep in the insulating regime which has never been explored prior to the present work. Unexpected behavior was seen in the thinnest films. These anomalies, specifically, a state characterized by a positive $\mathrm{d} R / \mathrm{d} T$, accompanied by a non-linear and asymmetric $I-V$ characteristic, were present only in the initial stage of film growth. As more material is deposited, this unconventional state abruptly disappears. After a transition regime, the films were found to undergo an S-I transition observed previously.

The evolution of the transport properties with respect to film thickness at the initial stage of film growth is attributed to a structural transition from an amorphous to granular structure in these thinnest quench condensed $\mathrm{Bi}$ films. The positive $\mathrm{d} R / \mathrm{d} T$ found in the amorphous, precursor layers were proposed to result from the formation of high electron density droplets within the insulating electron glass background. Though this model can qualitatively account for the features seen in the conduction, the nature of the amorphous precursor phase needs to be further studied, both experimentally and theoretically.

\section{ACKNOWLEDGEMENTS}

We acknowledge useful discussions with X. Xie and W. $\mathrm{Wu}$, and technical assistance from Yu. Zadorozhny. This work is supported by the National Science Foundation through Grant DMR-9702661.

${ }^{1}$ A. I. Shal'nikov, Nature 142, 74 (1938).

${ }^{2}$ Y. F. Komnik, Sov. J. Low Temp. Phys. 8, 1 (1982).

${ }^{3}$ G. Bergmann, Phys. Rep. 27, 160 (1976).

${ }^{4}$ G. Bergmann, Phys. Rep. 101, 1 (1984).

${ }^{5}$ Y. Liu and A. M. Goldman, Mod. Phys. Lett. B 8, 277 (1994); and references therein.

${ }^{6}$ M. Pollak, Discuss. Faraday Soc. 50, 11 (1970).

${ }^{7}$ A. L. Efros and B. I. Shklovskii, J. Phys. C 8, L49 (1975); A. L. Efros, J. Phys. C 9, 2021 (1976). 
8 J. H. Davies, P.A. Lee, and T. M. Rice, Phys. Rev. Lett. 49, 758 (1982)

${ }^{9}$ J. H. Davies, P. A. Lee, and T. M. Rice, Phys. Rev. B 29, 4260 (1984).

${ }^{10}$ W. Xue and P. A. Lee, Phys. Rev. B 38, 9093 (1988).

11 J. Ruiz, M. Ortuño, and E. Cuevas, Phys. Rev. B 48, 10777 (1993).

12 A. Díaz-Sanchez, M. Ortuño, A. Pérez-Garrido, and E. Cuevas, Phys. Stat. So. B 218, 11 (2000).

${ }^{13}$ K. Binder and A. P. Young, Rev. Mod. Phys. 58, 801 (1986).

${ }^{14}$ D. Monroe, A. C. Gossard, J. H. English, B. Golding, W. H. Haemmerle, and M. A. Kastner, Phys. Rev. Lett. 59 1148 (1987); J. G. Massey and M. Lee, Phys. Rev. B 62 R13270 (2000); and references therein.

15 M. Ben-Chorin, et al, Phys. Rev. B 48, 15025 (1993); Z. Ovadyahu and M. Pollak, Phys. Rev. Lett. 79, 459 (1997); A. Vaknin, Z. Ovadyahu, and M. Pollak, Phys. Rev. Lett. 84, 3402 (2000); and references therein.

${ }^{16}$ C. J. Adkins, J. M. D. Thomas, J. W. Gardner, and A. J. McGeown, J. Phys. C 174633 (1984).

${ }^{17}$ G. Martinez-Arizala, D. E. Grupp, C. Christiansen, A. M. Mack, N. Markovic, Y. Seguchi, and A. M. Goldman, Phys. Rev. Lett. 78, 1130 (1997); G. Martinez-Arizala, C. Christiansen, D.E. Grupp, N. Markovic, A.M. Mack, and A.M. Goldman, Phys. Rev. B 57, R670 (1998).

${ }^{18}$ C. C. Yu, Phys. Rev. Lett. 82, 4074 (1999).

19 N. Markovic, C. Christiansen, D. E. Grupp, A. M. Mack, G. Martinez-Arizala, and A. M. Goldman, Phys. Rev. B 62, 2195 (2000).

${ }^{20}$ K. L. Ekinci and J. M. Valles, Jr., Phys. Rev. B 58, 7347 (1998); Phys. Rev. Lett. 82, 1518 (1999).

${ }^{21}$ M. G. Mitch, S. J. Chase, J. Fortner, R. Q. Yu, J. S. Lannin, Phys. Rev. Lett. 67, 875 (1991).

22 A. V. Danilov, S. E. Kubatkin, I. L. Landau, I. A. Parshin, and L. Rinderer, Phys. Rev. B 51, 5514 (1995); A.V. Danilov, S. E. Kubatkin, I. L. Landau, and L. Rinderer, J. Low. Temp. Phys. 103, 1 (1996).

${ }^{23}$ M. M. Rosario, Yu. Zadorozhny, and Y. Liu, Phys Rev. B 61, 7005 (2000).

${ }^{24}$ E. I. Levin and B. I. Shklovskii, Sov. Phys. Semicond. 18, $534(1984)$

${ }^{25}$ A. G. Zabrodskii and K. N. Zinov'eva, Zh. Éksp. Teor. Fiz. 86, 727 (1984). [Sov. Phys. JETP 59, 425 (1984)].

${ }^{26}$ H. M. Jaeger, D. B. Haviland, B. G. Orr, and A. M. Goldman, Phys. Rev. B 40, 182 (1989).

${ }^{27}$ W. Wu and P. W. Adams, Phys. Rev. Lett. 73, 1412 (1994).

${ }^{28}$ D. B. Haviland, H. M. Jaeger, B. G. Orr, and A. M. Goldman, Phys. Rev. B 40, 719 (1989).

${ }^{29}$ R. C. Dynes, J. P. Garno, and J. M. Rowell, Phys. Rev. Lett. 40, 479 (1978).

${ }^{30}$ C. J. Adkins, J. M. D. Thomas, and M. W. Young, J.Phys. C 13, 3427 (1980).

${ }^{31}$ B. G. Orr, H. M. Jaeger, and A. M. Goldman, Phys. Rev. B. 32, 7586 (1985).

32 E. Simanek, Sol. State Comm. 31, 419 (1979); Phys. Rev. B 25, 237 (1982).

${ }^{33}$ M. P. A. Fisher, Phys. Rev. Lett. 57885 (1986).

${ }^{34}$ B. Weitzel and H. Micklitz, Phys. Rev. Lett. 66, 385 (1991).

${ }^{35}$ D. S. Fisher and D. A. Huse, Phys. Rev. B 38, 386 (1988).
36 J. Shi, S. He, and X. C. Xie, Phys. Rev. B 60, R13950 (1999); S. He and X. C. Xie, Phys. Rev. Lett. 80, 3324 (1998).

37 S. V. Kravchenko and T. M. Klapwijk, Phys. Rev. Lett. 84, 2909 (2000), and references therein.

FIG. 1. The time response of the voltage to an applied current bias of $+20 \mathrm{pA}$ at $T=0.60 \mathrm{~K}$. The solid line represents a fit to $V(t)=V_{\mathrm{o}}\left(1-\exp \left[-(t / \tau)^{\alpha}\right]\right)$ with $V_{\mathrm{o}}=0.22 \mathrm{~V}, \tau=80 \mathrm{~s}$, and $\alpha=0.8$. A schematic of the sample configuration is shown in the inset.

FIG. 2. $I-V$ characteristic of quench condensed Bi at a thickness of $12.5 \AA$. The voltages given are the limiting values, with the offset voltage for $I=0$ subtracted for each current. The temperatures are $0.60 \mathrm{~K}, 0.80 \mathrm{~K}, 0.90 \mathrm{~K}, 1.20 \mathrm{~K}$, $1.40 \mathrm{~K}, 1.80 \mathrm{~K}, 2.00 \mathrm{~K}, 2.20 \mathrm{~K}, 2.40 \mathrm{~K}, 2.80 \mathrm{~K}$ and $4.00 \mathrm{~K}$. The first fully linear curve was found at $T \approx 2.2 \mathrm{~K}$. All resistance measurements taken deep in the insulating regime were made using a +20 pA current bias.

FIG. 3. The resistance as a function of temperature $\mathrm{R}(T)$ for a $12.5 \AA$ thick film, using a +20 pA current bias. A Zabrodskii plot of the data, shown in inset a), indicates that a hopping conduction exponent of $a=1 / 2$ best describes the resistance at higher temperatures. This dependence is best seen on a semilog plot of the $\mathrm{R}$ versus $\sqrt{T}$ as given in inset $\mathrm{b}$ ). The solid line is a fit to VRH with Coulomb interactions. The resistance deviated from this behavior at $T \approx 2.2 \mathrm{~K}$.

FIG. 4. The resistance characteristic at film thicknesses of a) $10.0 \AA$, b) $10.5 \AA$, c) $11.0 \AA$, and d) $11.5 \AA$. The lines are a guide to the eye.

FIG. 5. Thickness dependence of the $\mathrm{R}(T)$ for the $2.5 \mu \mathrm{m}$ long film. The dashed line denotes the temperature at which the resistance minima were found in the high-disorder limit. This temperature is seen to be close to the local $T_{\mathrm{c}}$ near the S-I transition.

FIG. 6. A semi-log plot of the sheet resistance $\mathrm{R}_{\square}$ at $T=8 \mathrm{~K}$ as a function of film thickness. The inset shows a proposed phase diagram for the amorphous to granular structural transition in quench condensed Bi films at the initial stage of film growth.

TABLE I. Contact resistance, $R_{\mathrm{c}}$, at various film thicknesses. Resistance values of the co-evaporated $35 \mu \mathrm{m}\left(R_{\text {long }}\right)$ and $2.5 \mu \mathrm{m}\left(R_{\text {short }}\right)$ films were compared at $T=8 \mathrm{~K}$. For films of thickness $11.0 \AA<d<15.5 \AA$, the film resistances were found to not scale and a value of the contact resistance could not be determined.

\begin{tabular}{clll}
\hline \hline thickness $(\AA)$ & $R_{\text {long }}(\Omega)$ & $R_{\text {short }}(\Omega)$ & $R_{\mathrm{c}}(\Omega)$ \\
\hline 9.5 & $2.50 \pm 0.05 \cdot 10^{11}$ & $2.00 \pm 0.05 \cdot 10^{10}$ & $2.3 \pm 0.9 \cdot 10^{9}$
\end{tabular}




\begin{tabular}{llll}
11.0 & $3.70 \pm 0.05 \cdot 10^{10}$ & $2.7 \pm 0.1 \cdot 10^{9}$ & $9 \pm 6 \cdot 10^{7}$ \\
12.0 & $1.36 \pm 0.05 \cdot 10^{10}$ & $8.4 \pm 0.1 \cdot 10^{7}$ & \\
12.5 & $5.8 \pm 0.1 \cdot 10^{9}$ & $7.0 \pm 0.5 \cdot 10^{6}$ & \\
13.0 & $9.8 \pm 0.2 \cdot 10^{8}$ & $5.2 \pm 0.1 \cdot 10^{5}$ & \\
14.0 & $5.60 \pm 0.05 \cdot 10^{6}$ & $2.35 \pm 0.05 \cdot 10^{4}$ & \\
14.5 & $5.8 \pm 0.5 \cdot 10^{5}$ & $1.4 \pm 0.1 \cdot 10^{4}$ & \\
15.5 & $2.3 \pm 0.2 \cdot 10^{5}$ & $6.6 \pm 0.1 \cdot 10^{4}$ & \\
17.5 & $2.91 \pm 0.05 \cdot 10^{4}$ & $2.59 \pm 0.05 \cdot 10^{3}$ & $5.4 \pm 0.9 \cdot 10^{2}$ \\
20.5 & $1.80 \pm 0.03 \cdot 10^{3}$ & $7.00 \pm 0.02 \cdot 10^{2}$ & $6.1 \pm 0.2 \cdot 10^{2}$ \\
22.5 & $9.73 \pm 0.05 \cdot 10^{2}$ & $4.17 \pm 0.05 \cdot 10^{2}$ & $3.74 \pm 0.06 \cdot 10^{2}$ \\
\hline
\end{tabular}




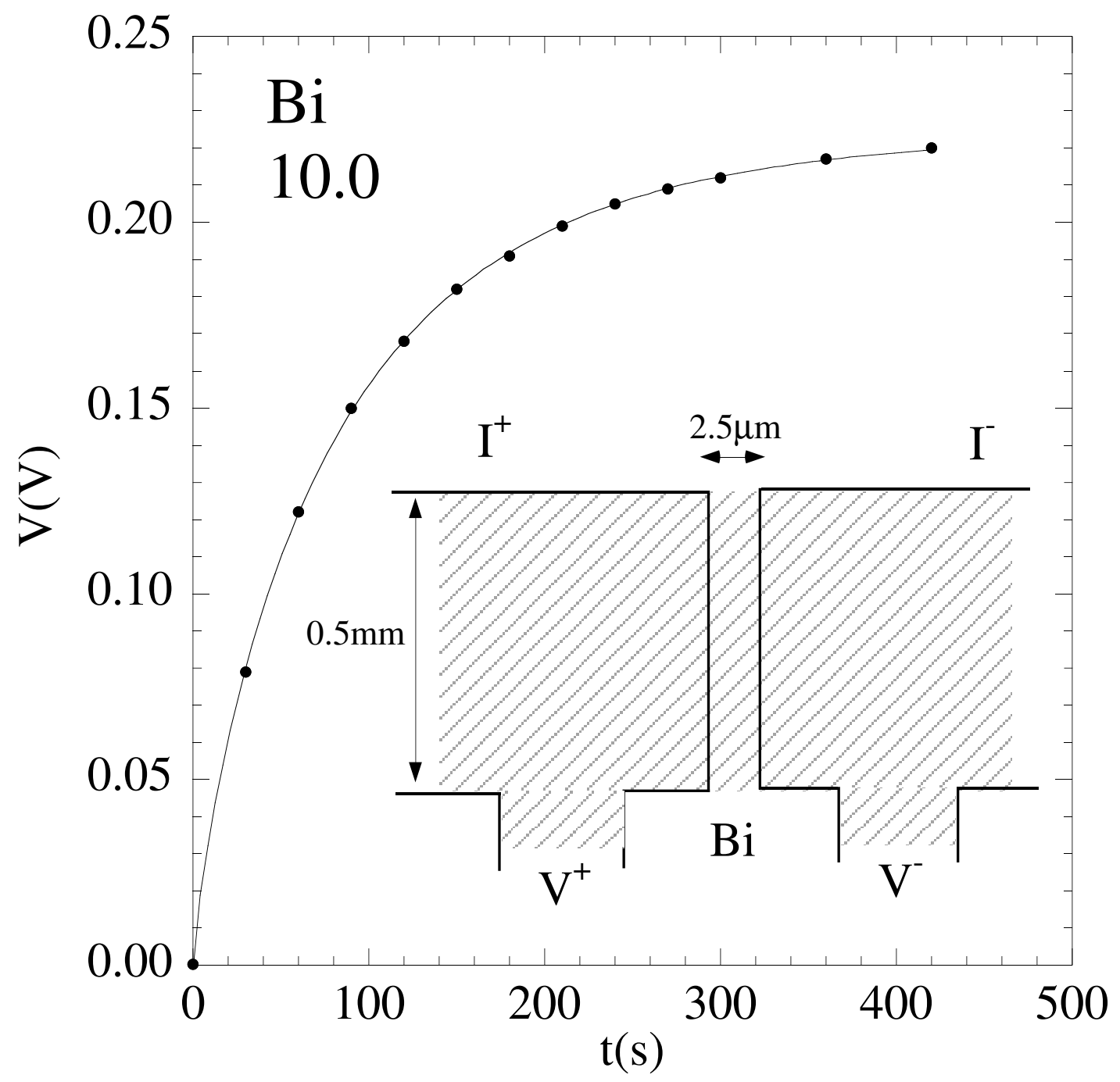

Fig. 1 M. M. Rosario and Y. Liu 


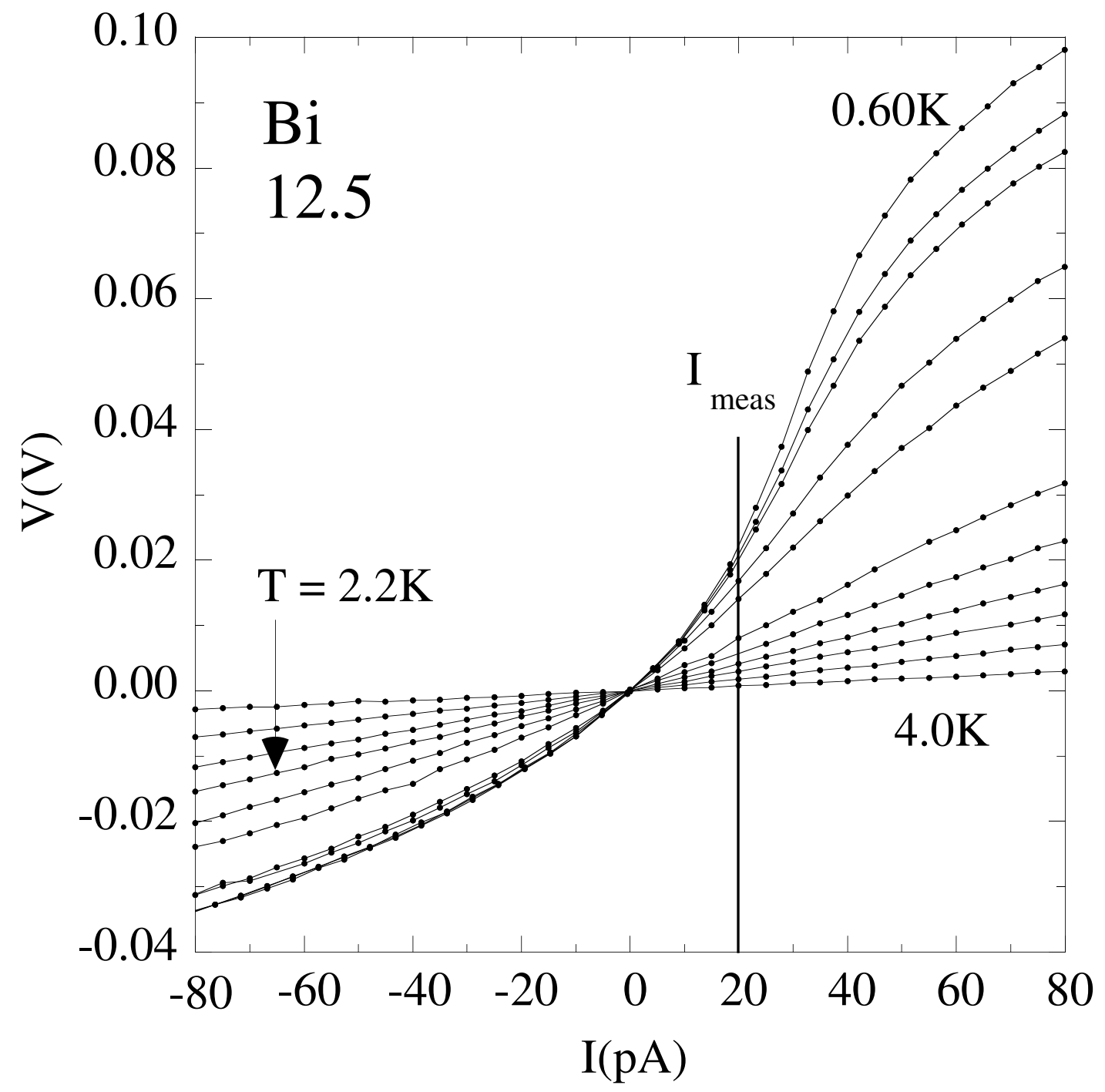

Fig. 2 M. M. Rosario and Y. Liu 


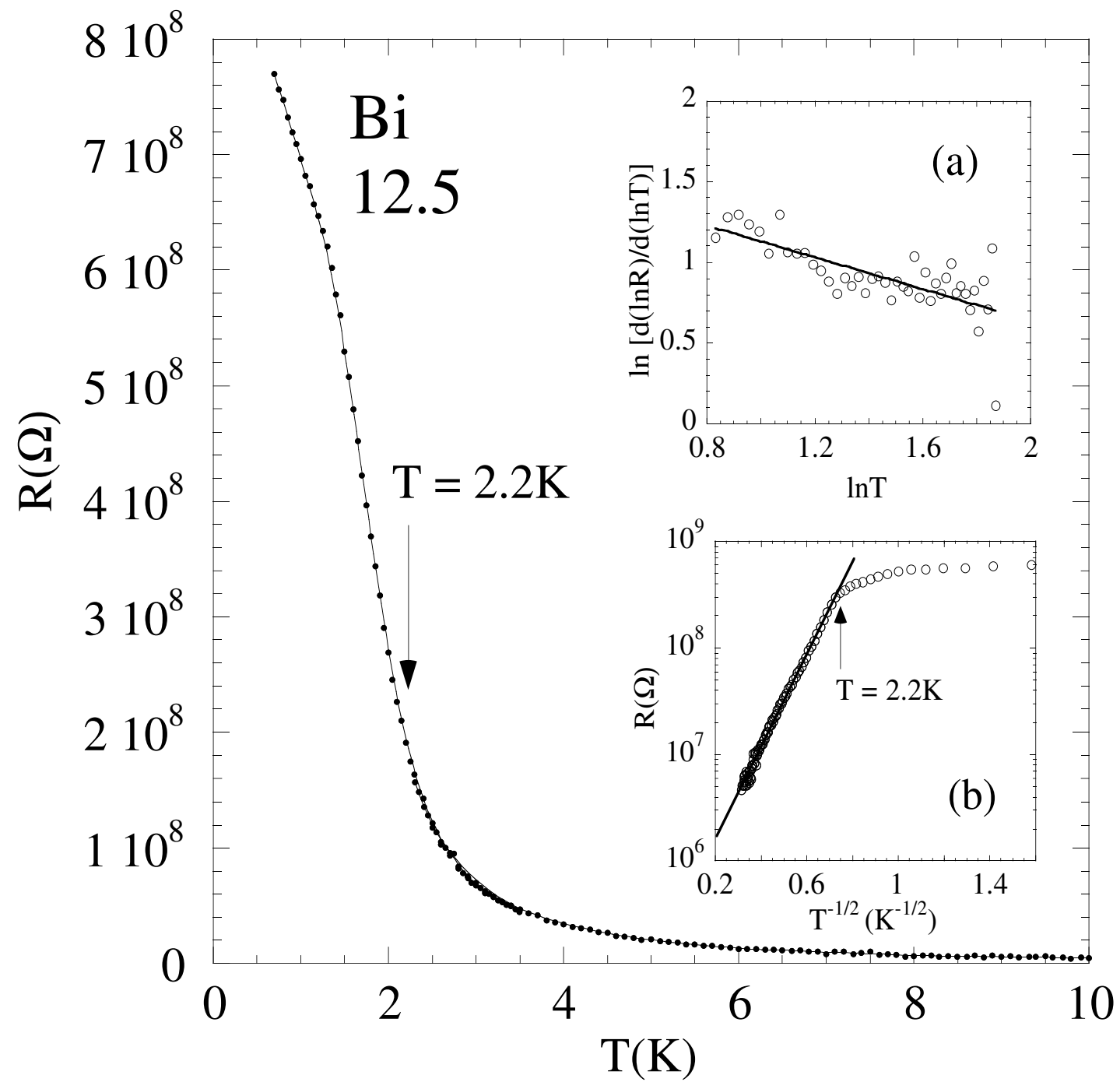

Fig. 3 M. M. Rosario and Y. Liu 

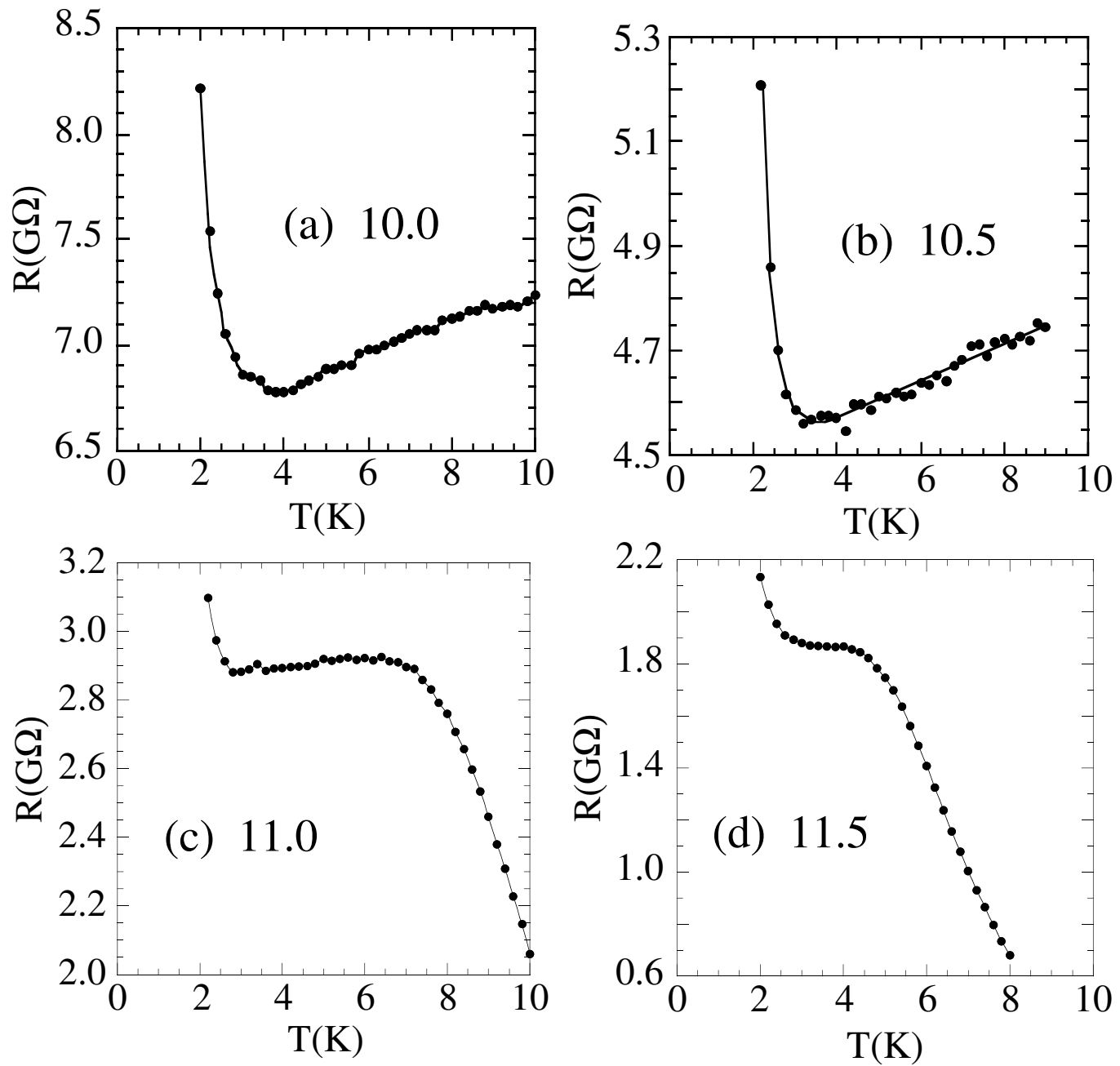

Fig. 4 M. M. Rosario and Y. Liu 


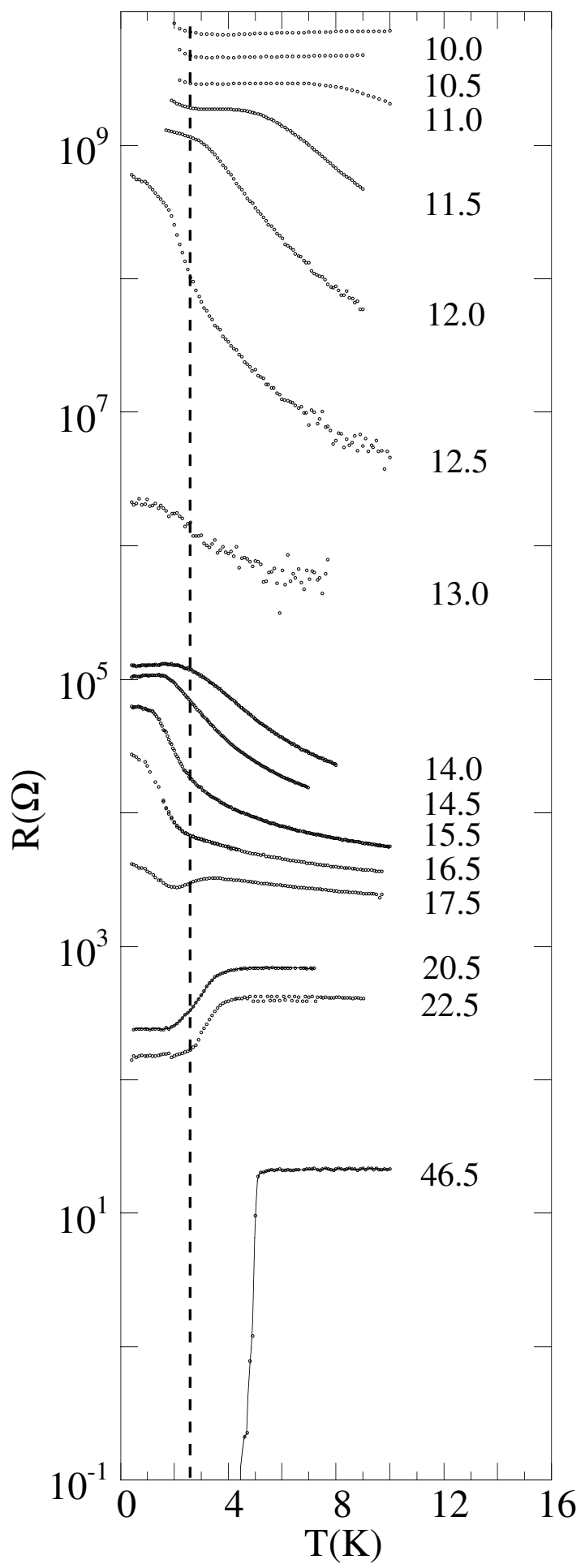

Fig. 5 M. M. Rosario and Y. Liu 


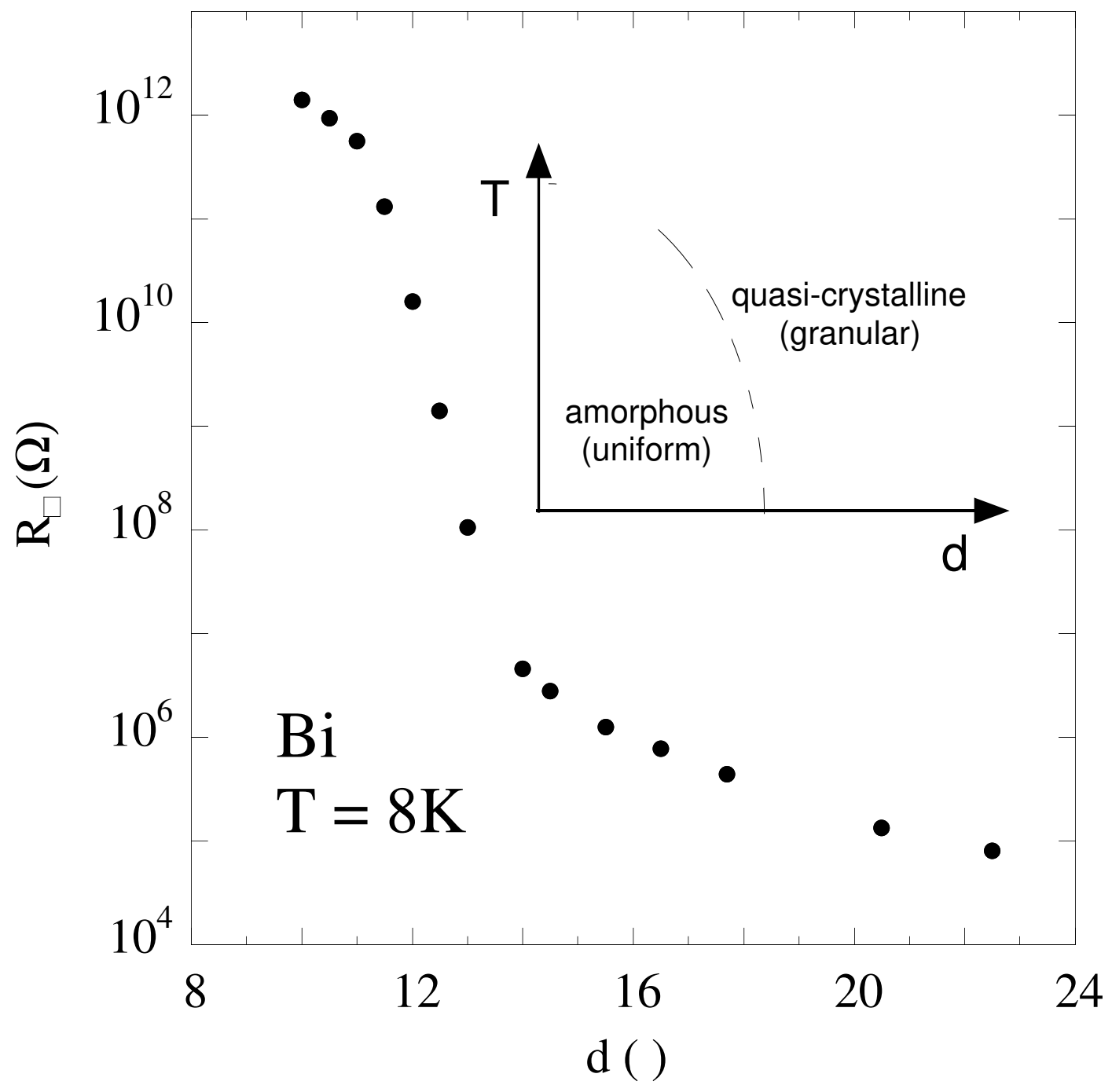

Fig. 6 M. M. Rosario and Y. Liu 